\title{
Validation of a low-cost portable 3-dimensional face scanner
}

\author{
Catherine Liu(Dי ${ }^{1, *}$, Andreas Artopoulos ${ }^{2}$ \\ ${ }^{1}$ Paediatric Dentistry Department, Dental Centre, Ground Floor South Wing, St Thomas' Hospital, Westminster Bridge Road, London, UK \\ ${ }^{2}$ Academic Centre of Reconstructive Science, King's College London Dental Institute, Floor 20, Tower Wing, Guy's Hospital, Great Maze \\ Pond, London, UK
}

\section{ABSTRACT}

\begin{abstract}
Purpose: The goal of this study was to assess the accuracy and reliability of a low-cost portable scanner (Scanify) for imaging facial casts compared to a previously validated portable digital stereophotogrammetry device (Vectra H1). This in vitro study was performed using 2 facial casts obtained by recording impressions of the authors, at King's College London Academic Centre of Reconstructive Science.

Materials and Methods: The casts were marked with anthropometric landmarks, then digitised using Scanify and Vectra H1. Computed tomography (CT) scans of the same casts were performed to verify the validation of Vectra H1. The 3-dimensional (3D) images acquired with each device were compared using linear measurements and 3D surface analysis software.

Results: Overall, $91 \%$ of the linear Scanify measurements were within $1 \mathrm{~mm}$ of the corresponding reference values. The mean overall surface difference between the Scanify and Vectra images was $<0.3 \mathrm{~mm}$. Significant differences were detected in depth measurements. Merging multiple Scanify images produced significantly greater registration error.

Conclusion: Scanify is a very low-cost device that could have clinical applications for facial imaging if imaging errors could be corrected by a future software update or hardware revision.(Imaging Sci Dent 2019; 49: 35-43)
\end{abstract}

KEY WORDS: Imaging, Three-Dimensional; Stereophotogrammetry; Validation Studies

\section{Introduction}

Three-dimensional (3D) facial imaging is valuable for capturing the facial topography in contexts where conventional 2-dimensional (2D) photography conveys limited information. ${ }^{1}$ Its main applications include monitoring growth and soft tissue changes, surgical treatment planning (mostly in maxillofacial and orthognathic surgery), assessment of post-operative results, and construction of facial prostheses. $^{1-3}$

The current options for 3D facial imaging are either volumetric or surface imaging methods. Volumetric techniques include computed tomography (CT), cone-beam CT (CBCT), and magnetic resonance imaging (MRI). Whilst

*King's Undergraduate Research Fellowships Scheme provided funding for this study. Received October 10, 2018; Revised December 6, 2018; Accepted December 15, 2018 *Correspondence to : Dr. Catherine Liu

Paediatric Dentistry Department, Dental Centre, Ground Floor South Wing, St Thomas' Hospital, Westminster Bridge Road, London, UK, SE1 7EH.

Tel) 44-744-387-0265, E-mail) catherineliu.dr@gmail.com these techniques can capture internal and external anatomy, they incur very high costs and require qualified staff to operate, and CT and CBCT involve exposure to ionising radiation. Additionally, the patient must be supine for $\mathrm{CT}$ and MRI scans, potentially distorting the soft tissues. ${ }^{4}$ Thus, they are not appropriate for long-term follow-up of patients or when multiple images are required.

Optical imaging techniques (such as digital stereophotogrammetry, laser scanning, and structured light pattern methods) use visible light, and therefore are indicated for routine or repeated facial imaging, but only capture external structures. Soft tissue landmarks are considered less reproducible than hard tissue landmarks on 2D cephalometric images; ${ }^{5}$ however, good soft tissue outcomes are a major goal of orthodontic and maxillofacial treatment, and are especially likely to determine patient satisfaction. ${ }^{1,5}$ Therefore, it is important to have accurate and reliable methods of recording external facial data.

A widely-used technique for optical facial imaging is 3D 
digital stereophotogrammetry due to its ease of use and fast data acquisition time. ${ }^{6}$ Other advantages include high-quality surface data and texture information, as well as the ability to image the face in the anatomic head position. Because it is also safe and reproducible, ${ }^{5}$ it can be used for repeated measurements.

Various 3D digital stereophotogrammetry systems are available, such as Canfield (Canfield Scientific, Parsippany, NJ, USA), 3dMD (3dMD, London, UK) and Di3D (Dimensional Imaging, Glasgow, Scotland, UK). These comprise multiple camera and flash units that require careful setup, calibration, and dedicated floor space, and range in price from approximately $£ 25,000$ to $£ 35,500$. More recently, portable, single-camera stereophotogrammetry devices have become available, such as the Vectra H1 (Canfield Scientific, Parsippany, NJ, USA), which has been previously validated $^{3}$ and is marketed in the region of $£ 13,000$. The advantages of a portable system are ease of use in any clinical setting with the subject in any position, easy transfer and storage, and minimal training requirements.

Another portable scanner, Scanify (Fuel 3D Technologies, Chinnor, UK) was launched in 2015 as an ultra-lowcost, handheld optical scanner based on 3D stereophotogrammetry, suitable for 3D capturing of the face. This scanner was available commercially for $£ 950$ until July 2017. However, there is a lack of evidence in the scientific literature on the level of accuracy and precision that can be achieved with such a low-cost device, and whether the obtained images could meet clinical requirements.

The aim of this study was to validate the Scanify device for 3D imaging of facial casts. Our null hypotheses were that there would be no significant differences when comparing linear measurements between facial landmarks on scans of facial casts acquired with Scanify and Vectra H1, and that there would be no significant overall surface deviations when comparing 3D surface models generated from scans of facial casts acquired with Scanify and Vectra H1.

\section{Materials and Methods}

Two facial gypsum casts were obtained from irreversible hydrocolloid impressions of both authors (labelled sample 1 and sample 2). Thirteen anthropometric landmarks ${ }^{7,8}$ were placed on each cast using a size 3 steel rosehead bur and black ink. The casts were digitised using the Scanify device and a validated portable 3D digital stereophotogrammetry device (Vectra H1). ${ }^{3}$ The images produced by Vectra $\mathrm{H} 1$ were used as references to which the Scanify images were compared. The 3D images were assessed using linear mea-

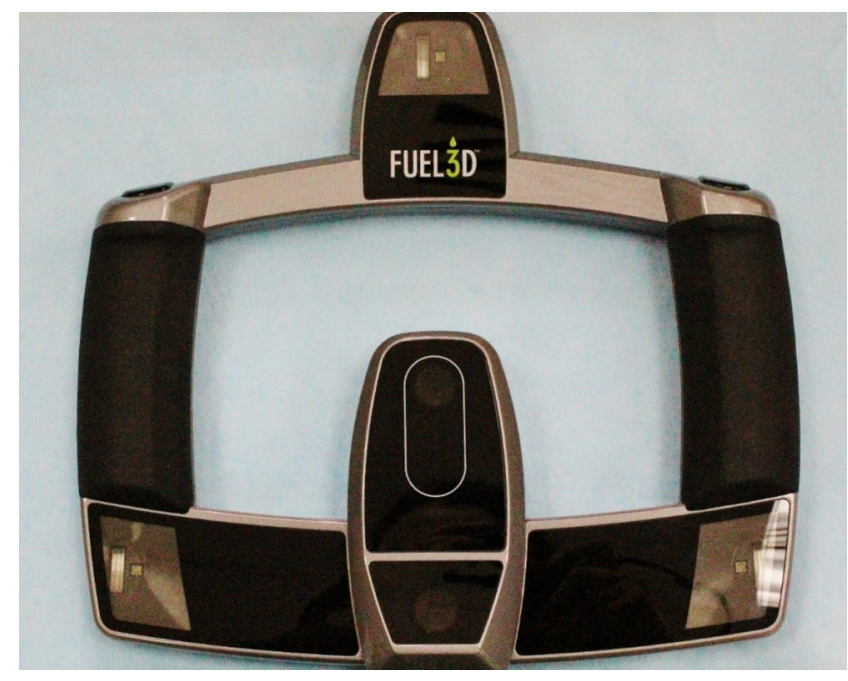

Fig. 1. The experimental device, Scanify (Fuel 3D Technologies, Chinnor, UK).

surements and 3D surface analysis.

The mean differences between linear measurements carried out directly on the casts and measurements carried out on 3D images were calculated. Bland-Altman limits of agreement were calculated to compare the linear measurements obtained using each method.

\section{The devices}

The Scanify device, shown in Figure 1, is an optical scanner that combines 3D stereophotogrammetry and stereophotometry. The device is lightweight at $0.51 \mathrm{~kg}$, mains-powered, and tethered to a computer. The Fuel3D Studio Software (Fuel 3D Technologies, Chinnor, UK) enables basic software operation, editing, and exporting functions.

In this device, two 3.5-megapixel RGB cameras are positioned at an obtuse angle to each other. Three xenon flash lights are triggered sequentially within milliseconds, illuminating the object at different angles and thereby allowing the cameras to capture 3 images of different shadows generated on the object surface. The images are then processed to reconstruct a 3D image. The data acquisition speed is 0.1 seconds per scan, followed by a flash cool-down period of 15 seconds. The field of view is $210 \mathrm{~mm} \times 300 \mathrm{~mm}$, and the operating range is $350-450 \mathrm{~mm}$.

Both the experimental and reference device (Vectra H1) are pre-calibrated by their manufacturers; however, Scanify requires an optical tracking target to be positioned close to the object. This enables the software to compensate for any motion during the capture and provides a reference pattern for merging multiple captures. 

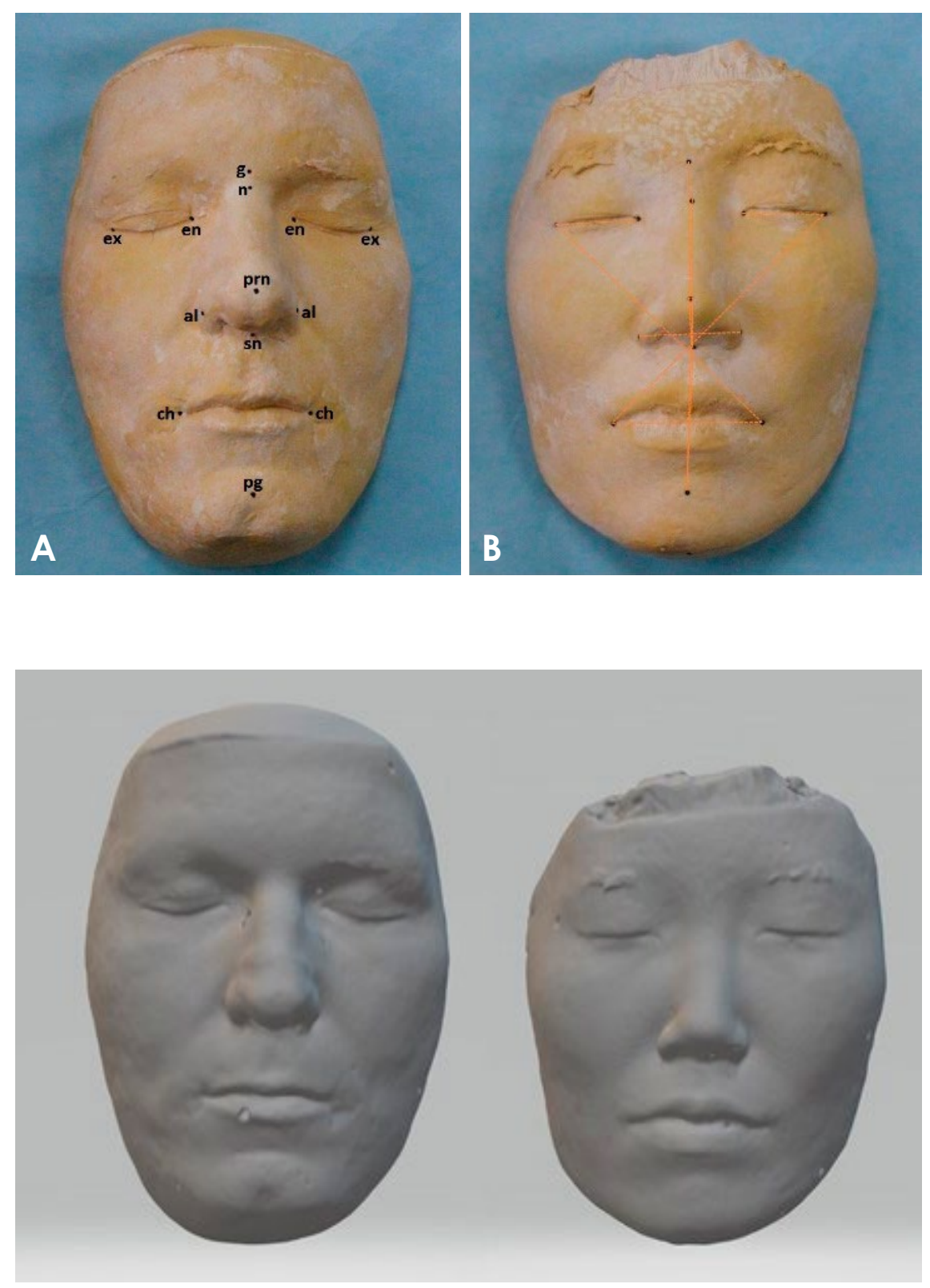

Fig. 2. A. Facial cast (sample 1) illustrating the 13 anthropometric landmarks placed on each cast. B. Facial cast (sample 2) illustrating the 11 inter-landmark measurements that were performed.

Fig. 3. Frontal views of computed tomography scans of both facial casts.
The Vectra $\mathrm{H} 1$ is a passive 3D digital stereophotogrammetry scanner. It uses a Canon Rebel T6i camera body and is battery-operated. It deploys 2 ranging lights that converge on the target object when the camera is held at the optimum distance of $450-500 \mathrm{~mm}$. It has a geometric resolution of $0.95 \mathrm{~mm}$ and captures 2 images simultaneously at an angle by using mirrors within a lens attachment. ${ }^{3}$ According to the manufacturer, it has a 2-millisecond capture time, and a capture volume of $270 \mathrm{~mm} \times 165 \mathrm{~mm} \times 100$ $\mathrm{mm}$. The Vectra H1 comes with the manufacturer's software (Mirror software and VECTRA analysis module; Canfield Scientific, Parsippany, NJ, USA).

\section{Digitisation of the facial casts}

The casts were first imaged by a third-generation spiral CT scanner (Brilliance iCT, Philips Healthcare, Amsterdam, Netherlands) in a pilot study, as this is the gold standard in clinical 3D imaging. The findings corroborated those of the previous Vectra H1 validation study; therefore, the Vectra H1 images were used as a reference with which the Scanify images could be compared, in order to assess interchangeability between the 2 techniques.

The 2 facial casts were each imaged repeatedly using Scanify and Vectra H1. For each cast, 2 types of image were acquired using each device: 1 single-capture frontal view ('single-capture'), and 1 multiple-capture image pro- 


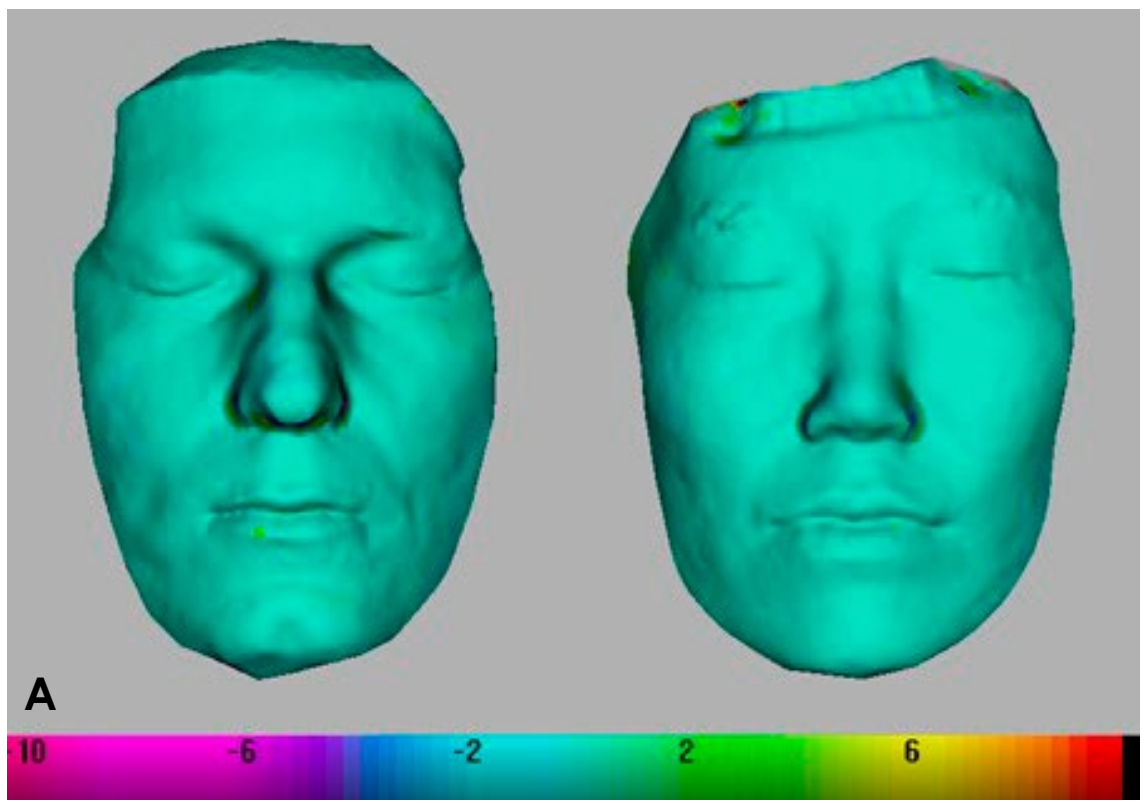

Fig. 4. Colour maps showing the minimal surface differences between the reference computed tomography scans and (A) single-capture Vectra images and (B) merged Vectra images, for both facial casts.

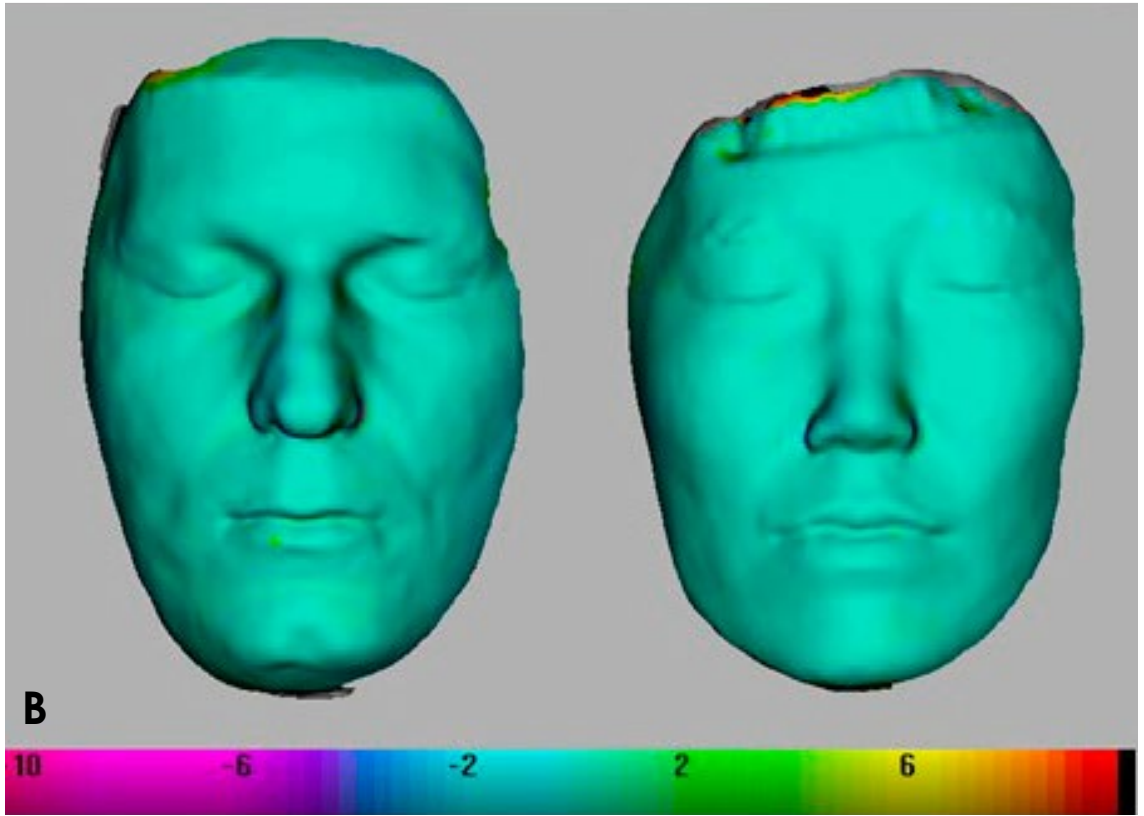

duced by merging 3 scans from different angles ('merged'). This technique of merging 3 images is recommended by the manufacturer when using Vectra $\mathrm{H} 1$ to capture the whole face, so the same protocol was applied using Scanify. ${ }^{3}$ The first scan was taken from directly in front of the cast at the level of the columella, and the second and third scans were taken from the left and right sides and below the level of the chin at $45^{\circ}$ from the long axis of the face. Each set of 3 images was then merged using 3D mesh manipulation software (Robins 3D, version 2.2.80, Robin Richards, London, UK). For each cast, forty 3D images were obtained with Scanify (20 single-capture and 20 merged).

\section{Linear measurements}

Inter-landmark measurements were performed on the 3D images using 3D mesh processing software (MeshLab, CNR-ISTI, Pisa, Italy). The same measurements were also performed directly on the casts using digital callipers to obtain reference values, as this method has been established in the literature. 9

The following landmarks were used, as illustrated in Figure 2A: glabella, nasion, pronasale, subnasale, pogonion, left and right exocanthion, left and right endocanthion, left and right alae, and left and right cheilion. Eleven inter-landmark measurements were recorded on each cast at various orientations in order to assess the accuracy of the 
Table 1. Comparisons between inter-landmark measurements taken by digital measurements of single-capture Scanify images and 3 different reference methods

\begin{tabular}{|c|c|c|c|c|c|c|}
\hline \multirow{2}{*}{$\begin{array}{c}\text { Facial cast } \\
\text { Reference method }\end{array}$} & \multicolumn{3}{|c|}{ Sample 1} & \multicolumn{3}{|c|}{ Sample 2} \\
\hline & Direct calliper & Single-capture Vectra & Merged Vectra & Direct calliper & Single-capture Vectra & Merged Vectra \\
\hline $\begin{array}{l}\text { Average difference } \\
\quad( \pm \mathrm{SD})(\mathrm{mm})\end{array}$ & $0.048( \pm 2.04)$ & $0.031( \pm 1.77)$ & $-0.013( \pm 1.80)$ & $0.011( \pm 0.53)$ & $-0.0012( \pm 0.54)$ & $-0.14( \pm 0.61)$ \\
\hline $\begin{array}{l}95 \% \text { limits of } \\
\text { agreement }(\mathrm{mm})\end{array}$ & -3.96 and 4.05 & -3.45 and 3.51 & -3.55 and 3.52 & -1.03 and 1.05 & -1.06 and 1.06 & -1.34 and 1.06 \\
\hline $\begin{array}{l}\text { Correlation } \\
\text { between difference } \\
\text { and reference }\end{array}$ & -0.24 & -0.27 & -0.26 & -0.40 & -0.38 & -0.17 \\
\hline
\end{tabular}

Table 2. Mean surface differences and percentage data points within clinical bounds of $\pm 0.5 \mathrm{~mm}$ and \pm 1 mm between experimental and reference data, obtained from the surface comparisons of 3-dimensional images

\begin{tabular}{|c|c|c|c|c|c|c|c|c|c|}
\hline \multirow[b]{2}{*}{ Comparison } & \multicolumn{3}{|c|}{ Sample 1} & \multicolumn{3}{|c|}{ Sample 2} & \multicolumn{3}{|c|}{ Both casts } \\
\hline & $\begin{array}{c}\text { Mean } \\
\text { distance } \\
(\mathrm{mm})\end{array}$ & $\begin{array}{c}\% \\
\text { points } \\
\text { within } \\
\pm 0.5 \mathrm{~mm}\end{array}$ & $\begin{array}{c}\% \\
\text { points } \\
\text { within } \\
\pm 1 \mathrm{~mm}\end{array}$ & $\begin{array}{c}\text { Mean } \\
\text { distance } \\
(\mathrm{mm})\end{array}$ & $\begin{array}{c}\% \\
\text { points } \\
\text { within } \\
\pm 0.5 \mathrm{~mm}\end{array}$ & $\begin{array}{c}\% \\
\text { points } \\
\text { within } \\
\pm 1 \mathrm{~mm}\end{array}$ & $\begin{array}{c}\text { Mean } \\
\text { distance } \\
(\mathrm{mm})\end{array}$ & $\begin{array}{c}\% \\
\text { points } \\
\text { within } \\
\pm 0.5 \mathrm{~mm}\end{array}$ & $\begin{array}{c}\% \\
\text { points } \\
\text { within } \\
\pm 1 \mathrm{~mm}\end{array}$ \\
\hline $\begin{array}{l}\text { Single-capture Scanify } \\
\text { versus } \\
\text { single-capture Vectra }\end{array}$ & 0.023 & $51.67 \%$ & $70.80 \%$ & 0.018 & $56.89 \%$ & $79.99 \%$ & 0.021 & $54.28 \%$ & $75.40 \%$ \\
\hline $\begin{array}{l}\text { Single-capture Scanify } \\
\text { versus merged Vectra }\end{array}$ & -0.079 & $55.08 \%$ & $72.99 \%$ & -0.100 & $69.11 \%$ & $86.03 \%$ & -0.090 & $62.10 \%$ & $79.51 \%$ \\
\hline $\begin{array}{l}\text { Merged Scanify } \\
\text { versus merged Vectra }\end{array}$ & 0.300 & $46.48 \%$ & $66.37 \%$ & 0.470 & $44.82 \%$ & $67.90 \%$ & 0.39 & $45.65 \%$ & $67.13 \%$ \\
\hline $\begin{array}{l}\text { Single-capture Scanify } \\
\text { versus CT }\end{array}$ & 0.144 & $53.80 \%$ & $69.53 \%$ & 0.050 & $75.43 \%$ & $88.96 \%$ & 0.095 & $64.15 \%$ & $78.82 \%$ \\
\hline $\begin{array}{l}\text { Merged Scanify } \\
\text { versus CT }\end{array}$ & 0.126 & $53.15 \%$ & $70.03 \%$ & -0.089 & $65.65 \%$ & $84.02 \%$ & -0.038 & $59.44 \%$ & $77.07 \%$ \\
\hline $\begin{array}{l}\text { Single-capture Vectra } \\
\text { versus CT }\end{array}$ & -0.019 & $98.41 \%$ & $99.14 \%$ & -0.003 & $98.54 \%$ & $99.31 \%$ & 0.011 & $98.48 \%$ & $99.23 \%$ \\
\hline $\begin{array}{l}\text { Merged Vectra } \\
\text { versus CT }\end{array}$ & 0.015 & $95.70 \%$ & $98.33 \%$ & 0.079 & $97.87 \%$ & $98.84 \%$ & 0.047 & $96.88 \%$ & $98.61 \%$ \\
\hline
\end{tabular}

CT: computed tomography

scanned images in all dimensions (Fig. 2B).

Twenty repeats of each measurement were performed both directly on the casts and digitally on a randomly-selected Scanify image to calculate intra-operator repeatability error. All scans, measurements, and comparisons were undertaken by 1 operator, who repeated each measurement 20 times over a period of 5 days.

\section{Three-dimensional analysis}

The 3D images acquired by Scanify, Vectra, and CT were compared following surface-based registration ${ }^{10}$ using Robins 3D software, employing the nearest vertical iterative closest point (ICP) registration. Images were first manually aligned to the relevant reference image as closely as possible, after which the software aligned and registered the entire surfaces of both images using an ICP algorithm and rigid transformation. The software generated quantitative and qualitative 3D analysis reports. Differences in shape were quantified as the distances between corresponding pairs of coordinate points on the 2 surfaces along the Z-axis, and illustrated as colour-coded deviation maps.

\section{Results}

\section{CT comparisons}

The Vectra images showed high concordance with the 


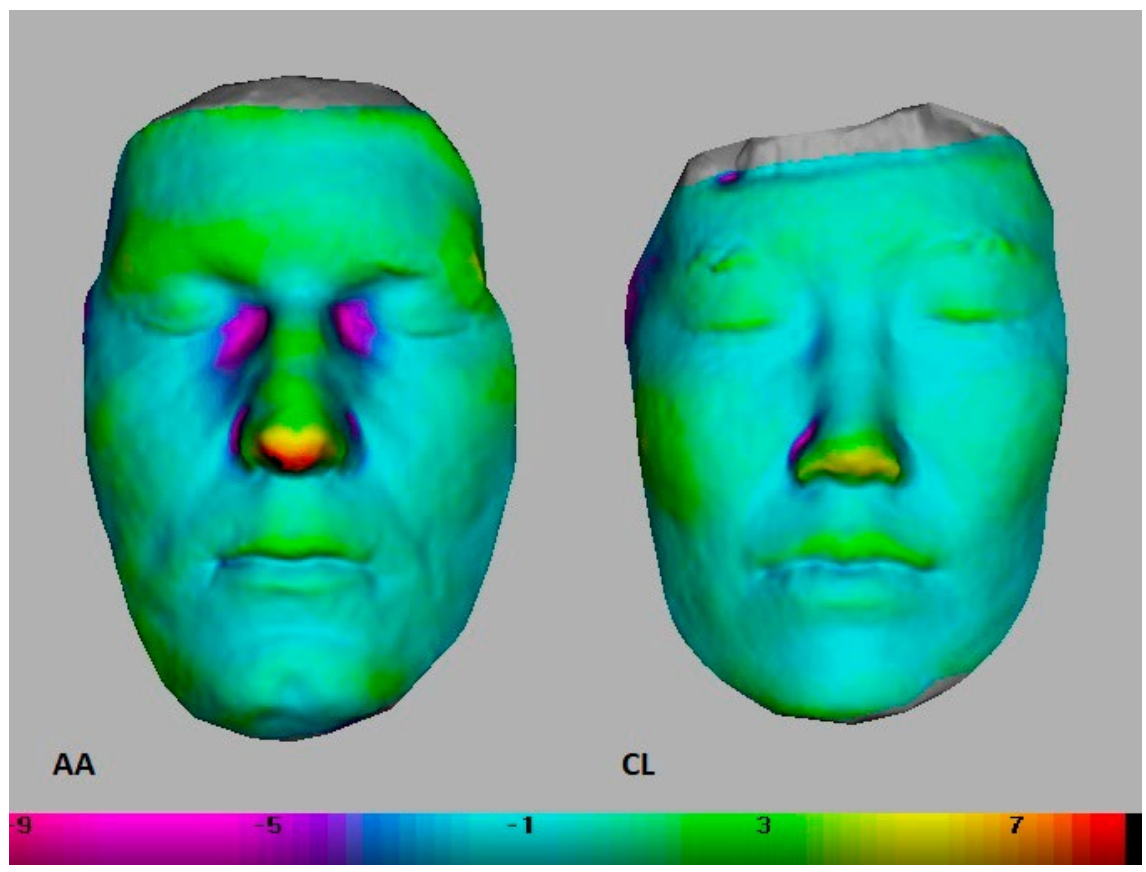

Fig. 5. Colour maps of both casts showing the surface deviations between the single-capture Scanify and single-capture Vectra $\mathrm{H} 1$ images in millimetres. Each pixel point was allocated a numerical value denoting the distance between corresponding points on the two 3 -dimensional images.

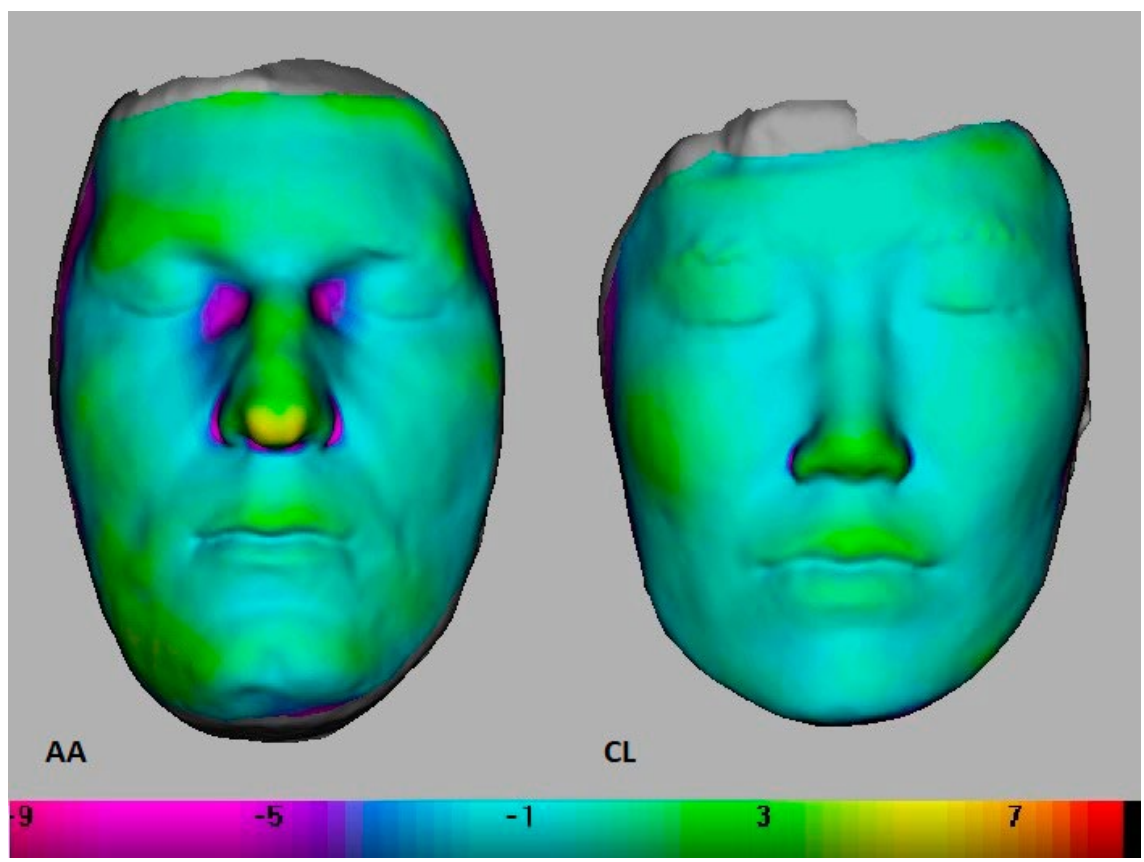

Fig. 6. Colour maps of both casts showing the surface differences between the single-capture Scanify and merged Vectra $\mathrm{H} 1$ images in millimetres.

CT images (Fig. 3): 99\% of data points on single-capture Vectra images and $98 \%$ of data points on merged Vectra images were within $1 \mathrm{~mm}$ of the corresponding points on CT scans for both casts. Figure 4 illustrates this in the form of colour maps.

\section{Inter-landmark measurements}

Linear measurements on single-capture Scanify images were compared to direct calliper measurements on the casts, measurements on single-capture Vectra images, and measurements on merged Vectra images. Data from the Bland-Altman plots are shown in Table 1.

The mean standard deviations of the direct calliper measurements, single-capture Scanify measurements, single-capture Vectra measurements, and merged Vectra measurements were $0.18 \mathrm{~mm}, 0.29 \mathrm{~mm}, 0.21 \mathrm{~mm}$, and 0.22 $\mathrm{mm}$ respectively. The mean coefficients of variation were $0.0041,0.0075$, and 0.0046 for the calliper measurements, 


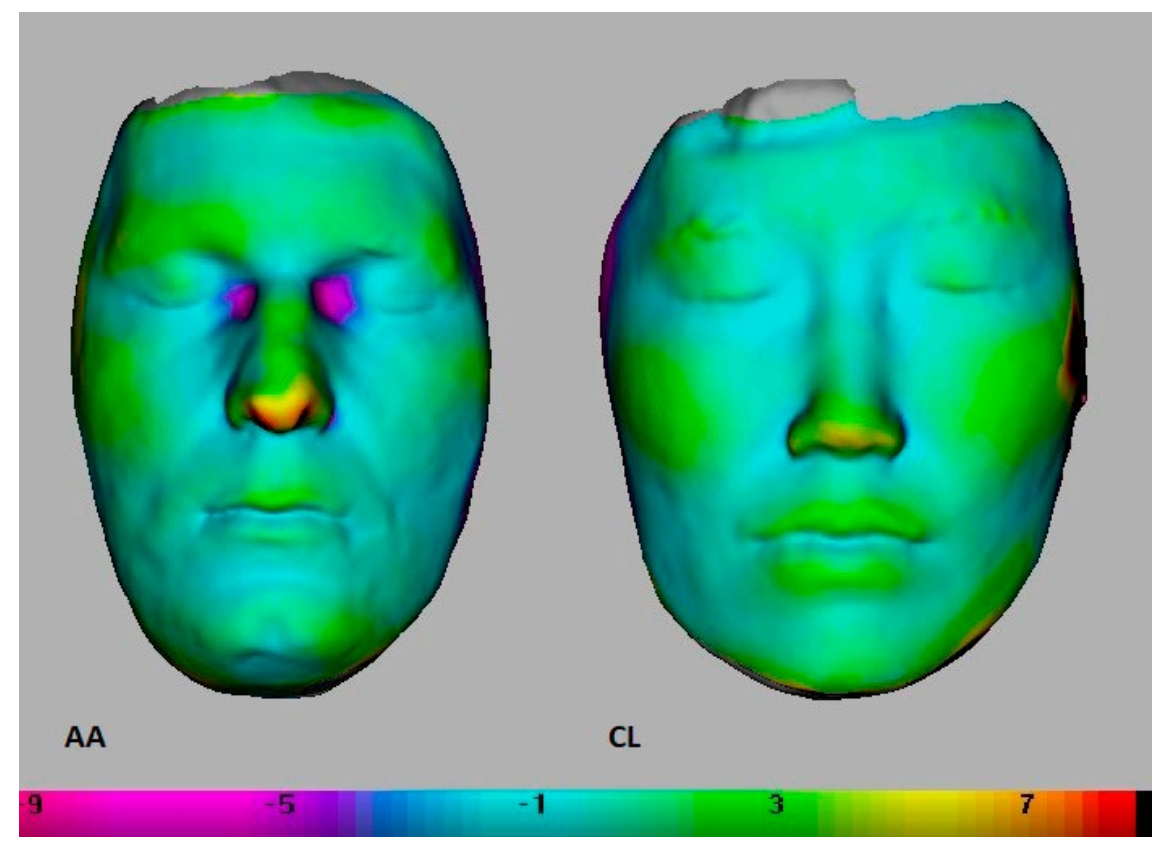

Fig. 7. Colour maps of both casts showing the surface differences between the merged Scanify and merged Vectra H1 images in millimetres.

Scanify measurements, and Vectra measurements, respectively.

\section{Three-dimensional surface comparisons}

Three sets of 3D surface comparisons were performed between the Scanify and Vectra images as follows: single-capture Scanify against single-capture Vectra, single-capture Scanify against merged Vectra, and merged Scanify against merged Vectra. Table 2 summarises the quantitative data from the 3D surface comparisons. Figures 5-7 show colour-coded maps representing the surface deviations between the 3D images taken by Scanify and Vectra.

\section{Registration error}

The mean registration error from merging the multiple single-capture scans was higher for Scanify $(0.74 \mathrm{~mm} \pm$ $0.089 \mathrm{~mm})$ than for Vectra $\mathrm{H} 1(0.15 \mathrm{~mm} \pm 0.015 \mathrm{~mm})$. There was no significant difference in the registration error between the 2 casts for the same device.

\section{Discussion}

The results of this study indicated that the Scanify images were comparable to the Vectra H1 images for simple topography, but showed significant errors in depth measurements.

Comparisons of inter-landmark measurements showed that the greatest differences between the Scanify and reference measurements were consistently of the subnasale-pro- nasale and nasion-pronasale distances, for both facial casts. A qualitative assessment of colour maps and quantitative data indicated that, although most data points on the Scanify images were within $\pm 1 \mathrm{~mm}$ of the Vectra data points, the greatest surface deviations (greater than $\pm 3 \mathrm{~mm}$ ) consistently occurred in areas of greater depth. For all comparisons, the mean differences between corresponding measurements and surface deviations were greater for sample 1 than for sample 2 . These findings suggest that the imaging error of Scanify was greatest along the z-axis.

The results also indicated increased error in merged images. Registration error should be sufficiently small as not to affect the interpretation of actual changes in facial morphology; Metzger et al. recommended a maximum of $1 \mathrm{~mm},{ }^{11}$ and the data from merged Scanify images in this study were within this limit. Merging multiple images allows a larger field of view, but also results in increased imaging error.

The limitations of in vitro testing apply to this study; gypsum casts were used as specimens to avoid variations due to motion artefacts and facial expression, so the results are likely to be more representative of the hardware's capabilities than of the findings that would be obtained when imaging living subjects. Conversely, the use of facial casts when comparing 3D scans to direct anthropometry avoids distortion of soft tissues under direct contact from callipers. ${ }^{1}$

The landmarks in this study were selected due to their previous use in similar studies, which affirmed their reli- 
ability as soft tissue anthropometric reference points; ${ }^{1,3,5,8}$ this also allowed comparison of the findings to the existing literature. The accuracy and reproducibility of most facial landmarks in 3D stereophotogrammetry has been well reported in the literature, ${ }^{5,9,12}$ with the least reproducible landmarks being the gonion, ${ }^{8}$ zygion, menton, and trichion. These are bony-related landmarks normally identified from radiographs, CT scans, or palpation; furthermore, the peripheral areas of the face show poorer illumination and contrast. ${ }^{13}$ These landmarks were not visible on the merged Scanify images due to loss of colour and surface detail during the merging process, and inter-landmark comparisons were therefore limited.

To the authors' knowledge, there have been no previous validation studies of the Scanify device with which our findings could be compared. In relation to other similar studies of common 3D stereophotogrammetric scanners, the findings of increased error in depth measurements appear to be unique to Scanify. Validation studies of Vectra H1 showed high accuracy and precision comparable to multi-camera systems, and few sites of complex shapes or peripheral points showed surface errors beyond the limits of clinical acceptability (greater than $\pm 1 \mathrm{~mm}$ ). ${ }^{3}$

Investigations of multi-camera systems are far more abundant than those of portable 3D stereophotogrammetric cameras. Most of these conclusions are in agreement in reporting acceptable accuracy, high reproducibility, good utility in longitudinal studies, and greater system error when imaging complex topography and peripheral structures. ${ }^{1,9,10,14}$ This highlights some general limitations of 3D optical imaging, including the loss of accuracy when capturing peripheral structures. ${ }^{2}$

Some alternatives to 3D stereophotogrammetry include manual anthropometric measurements, photocephalometry, moiré topography, 3D laser scanning, and structured light imaging. However, 3D stereophotogrammetry has largely superseded direct anthropometry (due to its time-consuming and unreliable nature) and photocephalometry (due to errors resulting from superimposition of the photographs and the corresponding cephalographs). ${ }^{15}$ Furthermore, 3D laser scanners take longer to scan an object than stereophotogrammetric devices, and the patient must keep his or her eyes closed throughout data capture to avoid the risk of damage from the laser beam. ${ }^{14}$

Since the completion of this study, the Scanify device has been discontinued. Nonetheless, other similar devices are still available on the market (for example Primesense Carmine 1.09 [Artcreation 3D Technology, Shanghai, China] and Gotcha [4D Dynamics, Antwerp, Belgium]), and more are likely to become available soon due to rapid technological developments in the field. This study highlights the value of low-cost, crowdfunded technology and the possible clinical applications of such devices in the future.

Within the limitations of this study, it was concluded that the Scanify device was comparable to Vectra $\mathrm{H} 1$ in accuracy and reliability on a millimetre scale when scanning a simple surface, but that it produced significant errors in depth measurements. The imaging errors from the Scanify device were within clinically acceptable levels for maxillofacial imaging in most areas of the face, but outside of the acceptable range in areas of greater depth measurements, such as the nose. Furthermore, the registration error from merging multiple images was greater for the experimental data than for the reference data.

Scanify was reliable in producing the same error for each measurement along the z-axis, suggesting that it could be used for comparative studies. This error could be corrected by using an appropriate algorithm incorporated into the image-processing software.

\section{Acknowledgements}

We would like to thank Professor Trevor Coward for hosting this study at the Academic Centre for Reconstructive Science.

\section{References}

1. Choi JW, Lee JY, Oh TS, Kwon SM, Yang SJ, Koh KS. Frontal soft tissue analysis using a 3 dimensional camera following two-jaw rotational orthognathic surgery in skeletal class III patients. J Craniomaxillofac Surg 2014; 42: 220-6.

2. Verhoeven TJ, Coppen C, Barkhuysen R, Bronkhorst EM, Merkx MA, Bergé SJ, et al. Three dimensional evaluation of facial asymmetry after mandibular reconstruction: validation of a new method using stereophotogrammetry. Int J Oral Maxillofac Surg 2013; 42: 19-25.

3. Artopoulos A, Rosenberg R, Coward TJ. Three-dimensional imaging of the face using a portable, single camera, digital stereophotogrammetry device. Poster session presented at: Optical Measurement Techniques for Systems and Structures (OPTIMESS) Meeting; 2015 April 8-9; Antwerp, Belgium.

4. Ozsoy U, Demirel BM, Yildirim FB, Tosun O, Sarikcioglu L. Method selection in craniofacial measurements: advantages and disadvantages of 3D digitization method. J Craniomaxillofac Surg 2009; 37: 285-90.

5. Plooij JM, Swennen GR, Rangel FA, Maal TJ, Schutyser FA, Bronkhorst EM, et al. Evaluation of reproducibility and reliability of 3D soft tissue analysis using 3D stereophotogrammetry. Int J Oral Maxillofac Surg 2009; 38: 267-73.

6. van Loon B, Maal TJ, Plooij JM, Ingels KJ, Borstlap WA, Kui- 
jpers-Jagtman AM, et al. 3D stereophotogrammetric assessment of pre- and postoperative volumetric changes in the cleft lip and palate nose. Int J Oral Maxillofac Surg 2010; 39: 534-40.

7. Farkas LG. Anthropometry of the head and face. 2nd ed. New York: Raven Press; 1994.

8. Gwilliam JR, Cunningham SJ, Hutton T. Reproducibility of soft tissue landmarks on three-dimensional facial scans. Eur J Orthod 2006; 28: 408-15.

9. Krimmel M, Kluba S, Dietz K, Reinert S. Assessment of precision and accuracy of digital surface photogrammetry with the DSP 400 system. Biomed Technol (Berl) 2005; 503: 45-53.

10. Maal TJ, Verhamme LM, van Loon B, Plooij JM, Rangel FA, Kho A, et al. Variation of the face in rest using 3D stereophotogrammetry. Int J Oral Maxillofac Surg 2011; 40: 1252-7.

11. Metzger MC, Hohlweg-Majert B, Schön R, Teschner M, Gellrich NC, Schmelzeisen R, et al. Verification of clinical precision after computer-aided reconstruction in craniomaxillofacial sur- gery. Oral Surg Oral Med Oral Pathol Oral Radiol Endod 2007; 104: e1-10.

12. Swennen GR, Schutyser F, Lemaitre A, Malevez C, De Mey A. Accuracy and reliability of 3-D CT versus 3-D stereo photogrammetry based facial soft tissue analysis. Int J Oral Maxillofac Surg 2005; 34: 73 (Abstr.).

13. Hajeer MY, Ayoub AF, Millett DT, Bock M, Siebert JP. Three-dimensional imaging in orthognathic surgery: the clinical application of a new method. Int $\mathbf{J}$ Adult Orthodon Orthognath Surg 2002; 17: 318-30.

14. Germeç-Çakan D, Canter HI, Nur B, Arun T. Comparison of facial soft tissue measurements on three-dimensional images and models obtained with different methods. J Craniofac Surg 2010; 21: 1393-9.

15. Phillips C, Greer J, Vig P, Matteson S. Photocephalometry: errors of projection and landmark location. Am J Orthod 1984; 86: 233-43. 